\title{
ESTADO DE LA TELEFONÍA EN EL PERÚ AL AÑO 2005
}

\author{
Augusto Hidalgo Sánchez* \\ E-mail: ahidalgos@unmsm.edu.pe
}

\begin{abstract}
RESUMEN
La telefonía en el Perú ha crecido de 3.4 a 28.8 teléfonos por cada cien habitantes en el periodo 19942005. Este crecimiento se ha dado dentro del marco de privatización de estos servicios públicos ocurrido en 1994, y dentro de las tendencias internacionales de incorporación de tecnologías de transmisión de alta velocidad, de caída de los costos y aumento inédito en la frecuencia de llamadas internacionales. Sin embargo, este fenómeno no es exclusivo del Perú, sino que abarca a toda América Latina. Más aún, el Perú resulta ser el país de menor desarrollo telefónico absoluto de la región, escenario donde resalta Chile con una densidad de 66 teléfonos por cada cien habitantes. Internamente existe una enorme concentración del servicio telefónico en Lima, con una densidad de 56 teléfonos por cada cien habitantes, mientras que en las regiones de la sierra y la selva la densidad es mucho menor, como en Huancavelica donde la densidad es de 1.6 teléfonos por cada cien habitantes. Frente a este panorama es necesario observar tanto el modelo de concesiones peruano como la poca actividad del Estado en la promoción de este servicio en las zonas más pobres del país.
\end{abstract}

Palabras claves: Telefonía Perú, celulares, penetración telefónica, Telefónica del Perú.

\section{ABSTRACT}

The telephonic service in Peru has grown from 3.4 to 28.8 lines per one hundred people in the period from $1994-2005$. This growing taked place in the setting of privatization norms of the public services occured in 1994, in middle of the international tendencies of incorporating high speed transmission technologies, cost reductions and the suddenly increase in the frecuency of international calls. Nevertheless, this phenomenon is not exclusive of Perú, but covers all Latin America. In addition, Perú has the least regional telephonic development, scene where Chile stands out with a density of 66 telephones per one hundred people. Internally, there is a highly telephonic service concentration in the city of Lima with a density of 56 lines per one hundred habitants, while regions in the highlands and jungle the density are 1.6 lines per one hundred habitants. Facing this scene is necessary to notice Peruvian concession model and also the small State activity in promoting this service in the poorest zones of the country.

Key Words: Peruvian Telephony, mobiles, telephone penetration, Telefónica del Perú.

Master of Science y MBA. Licenciado en Administración. Profesor de la Facultad de Ciencias Administrativas (UNMSM), Consultor en asuntos de comunicaciones y tecnologías de información. 


\section{INTRODUCCIÓN}

El Perú ha tenido una mínima penetración telefónica hasta comienzos de la década de los 90. Es a partir de entonces y hasta el año 2006, que está experimentando un aumento vertiginoso en el uso de los teléfonos.

Pero, ¿cuáles son las tendencias en la telefonía internacional?, ¿cuál es la magnitud de este crecimiento en los teléfonos fijos, en los celulares, en la telefonía a larga distancia y en la telefonía pública?, ¿cuáles son los factores que están propiciando este desarrollo?, ¿cómo se aplica a las diferentes ciudades y regiones del país?, ¿quiénes manejan la telefonía en el Perú?, comparativamente ¿qué está sucediendo en materia telefónica en otros países de la región latinoamericana y del mundo?, ¿qué restricciones enfrenta el desarrollo telefónico peruano? Estas son algunas de las interrogantes que se tratan de responder en el presente reporte de investigación, que ha sido elaborado sobre la base de información electrónica publicada en las páginas web oficiales de diversas instituciones públicas y privadas, nacionales y extranjeras.

\section{MÉTODO}

El método seguido fue de monitoreo electrónico. Se organizó una base de datos que fue nutrida trimestralmente por estadísticas oficiales de diversas instituciones y empresas del Perú y del extranjero durante los últimos dos años. El procesamiento de los datos ha sido porcentual.

\section{TENDENCIAS EN LA TELEFONÍA INTERNACIONAL}

El teléfono se ha vuelto un medio de comunicación de uso cada vez mayor tanto a nivel de los negocios como de las personas en todo el mundo. Tal vez la demostración más contundente e inaudita de esta afirmación es la tasa de crecimiento de la telefonía en la República Popular China en el año 2005. Según estadísticas gubernamentales, el país llegó a tener funcionando 351 millones de teléfonos residenciales fijos y 388 millones de teléfonos celulares, lo que da una densidad de 56.8 teléfonos por cada cien habitantes y a un ritmo de crecimiento de $15 \%$ al año ${ }^{1}$; o sea que solamente en el año 2005, dicho país ha crecido en 110 millones de aparatos telefónicos entre residenciales y celulares, cantidad que resulta mayor que todos teléfonos con los que cuenta Inglaterra.
Pero, aparte de este fenómeno de impacto mundial del gigante asiático, existen tres tendencias en el mercado internacional de telefonía, relacionadas entre sí, y que permiten identificar las causas y también dimensionar los efectos.

\section{Aumento de las llamadas telefónicas}

Las tendencias en el uso del teléfono son más fáciles de comprender si analizamos en el largo plazo el fenómeno en un país desarrollado. Para esto, vamos a tomar el caso de Estados Unidos y basarnos en las cifras que publica la Federal Communications Commission (FCC), organismo regulador de las telecomunicaciones en dicho país que ha sido elegido para este estudio por ofrecer estadísticas confiables de largo plazo.

Comencemos por las llamadas internacionales (Ver Gráfico 1). Precisamente según la FCC, las llamadas internacionales salientes de dicho país arrojan un crecimiento explosivo, pasando de 200 millones de llamadas en 1980 a 5,900 millones en el 2002. Esto representa un aumento de 30 veces en 22 años.

Esta alza en el número de llamadas es refrendada por el aumento de casi tres veces en el gasto telefónico de la familia promedio. Dicho gasto subió en dólares constantes de US\$ 360.00 en 1981 a US\$ 957.00 en el presupuesto familiar del año $2002^{2}$.

\section{Gráfico 1}

Llamadas salientes desde Estados Unidos.

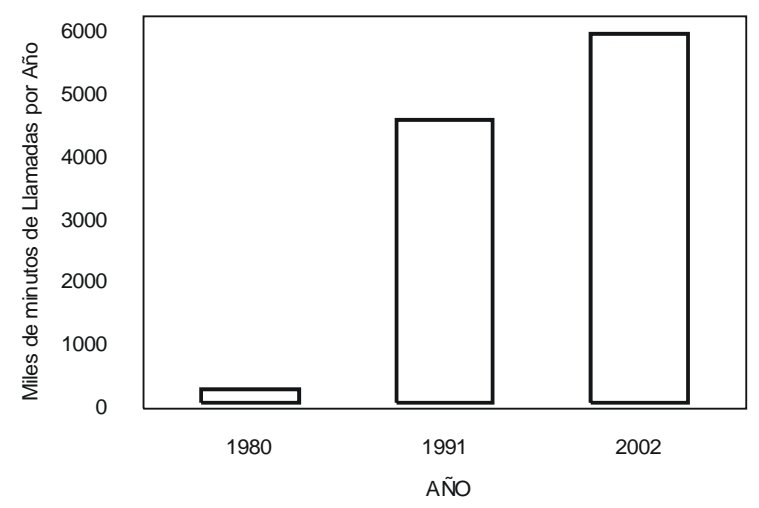

Mejora en la tecnología de transmisión y conexión telefónica

Otra tendencia que se observa en el mundo es la mejora de la tecnología utilizada para la transmisión y conexión telefónica. Concretamente, a partir de los años 90, se observa el uso masivo de la fibra óptica en vez del tradicional cable de cobre. La fibra óptica brinda una capacidad de transmisión 30 mil veces mayor que el cobre en 
promedio. Este cambio tecnológico de líneas relativamente lentas a líneas de alta velocidad de transmisión ha conducido a un aumento casi vertical de la oferta de servicios.

Precisamente, de acuerdo a cifras oficiales del gobierno norteamericano, observamos que las conexiones telefónicas de alta velocidad a los hogares han crecido de 1,8 millones en 1999 a 20,7 millones en el 2003. Es decir, ha crecido doce veces en solo cuatro años.

La tecnología que ha despuntado es la combinación de fibra óptica con el cable coaxial; la primera para conducir la señal por miles de kilómetros y la segunda para «la última milla», es decir hasta el hogar. Esta tecnología mixta ha probado además ser sumamente práctica, ya que por el mismo cable es posible transmitir una amplia variedad de servicios de comunicaciones como: conversaciones telefónicas, mensajería, canal del tiempo, canales de cable de video, acceso a Internet de banda ancha, etc.; todo en una sola conexión.

La masificación de la fibra óptica a nivel mundial en los años 90 ha desplazado a un lejano segundo plano el uso del satélite para las comunicaciones telefónicas internacionales. El satélite, un medio caro y prohibitivo, ha sido convertido esencialmente en un recurso tecnológico de respaldo, de urgencia, o de «redundancia» o ha quedado para ser utilizado por clientes exclusivos, pero, ya no es más el medio común u ordinario para transmitir mensajes telefónicos, como lo era en los 80. Por lo tanto, las comunicaciones a partir de los 90 ya no dependen más del escaso recurso satelital, que era propiedad exclusiva de los países desarrollados. Recordemos que el Perú, por ejemplo, no tiene un solo satélite en órbita.

\section{Costo por minuto de las llamadas telefónicas}

Siguiendo la ley de la oferta y la demanda, el aumento vertical de la oferta ha causado una reducción casi traumática en el costo del minuto de cada llamada. Esta baja en el costo por minuto de llamada promedio es el otro fenómeno relacionado que se observa en el contexto internacional. La baja en tarifas incluye llamadas locales, nacionales e internacionales.

Tomemos una vez más el caso de los Estados Unidos (Ver Gráfico 2). Luego de recoger y analizar las series estadísticas que se tienen desde 1930 a la fecha, descubrimos que el costo de la llamada combinada promedio ha descendido de US\$ 2.96 el minuto en 1930 a US\$ 0.09 en el 2002 , en una tendencia decreciente invariable, lo que significa que en los últimos setenta años el costo de la llamada en dicho país ha bajado en 33 veces.

\section{Gráfico 2}

Evolución del costo por minuto de las llamadas.

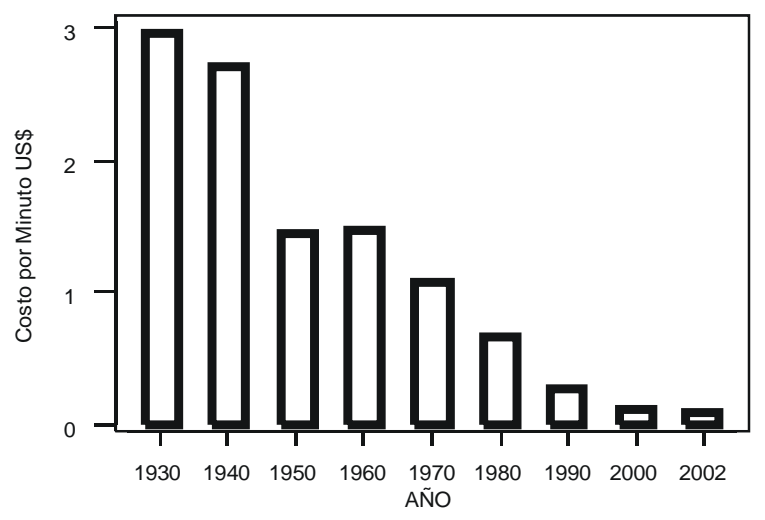

El costo de la llamada internacional en los últimos veinte años también ha descendido, igualmente. Según cifras de la FCC, el costo para la llamada saliente de los Estados Unidos ha bajado de 17.26 centavos en 1984 , a solamente 1.44 centavos el minuto en el 2003, lo que representa una baja de doce veces en un periodo de veinte años. Este descenso es proporcionalmente mayor que el costo de la llamada combinada promedio, debido a que ocurrió en solamente diecinueve años.

\section{SITUACIÓN Y TENDENCIAS DE LA TELEFONÍA EN EL PERÚ}

Con el panorama de tendencias descrito ahora podemos ingresar a analizar el caso peruano. Hasta hace una década, tener servicio telefónico en el hogar peruano era un lujo, y un signo de status social. A fines de los 80 la instalación de un teléfono residencial costaba un promedio de US\$ 1,200.00 por una línea. La telefonía pública también era muy escasa, correspondientemente. El servicio era atendido por dos compañías estatales: Compañía Peruana de Teléfonos (CPT) para la atención al mercado de Lima, y la Empresa de Telecomunicaciones del Perú (Entel Perú) para el servicio nacional restante.

Pero a partir de 1994, año en el que el gobierno privatizó el servicio tanto a nivel de Lima como a nivel nacional ${ }^{3}$, el Perú viene experimentando un crecimiento sostenido en la densidad telefó- 
nica. Según cifras oficiales publicadas por el Organismo Supervisor de la Inversión Privada en Telecomunicaciones del Perú (OSIPTEL), el país ha pasado de 3.43 teléfonos por cada cien habitantes en 1994 a 28.8 en el 2005 (Ver Gráfico 3), lo que, en cifras relativas, resulta un salto espectacular de siete veces en once años. (Ver Cuadro 1).

Esta mayor disponibilidad de teléfonos se observa en los tres tipos de servicios telefónicos: en fijos residenciales, en móviles de tipo celular y en los teléfonos públicos. En los teléfonos fijos este crecimiento significó pasar de 760 mil teléfonos en 1994 a 2 millones 250 mil, en el 2005, lo que representa un crecimiento de tres veces en once años. Complementariamente, el crecimiento del teléfono celular en el mismo periodo avanzó desde 52,000 celulares hasta 5 millones y medio; es decir, 107 veces en el mismo periodo.

El efecto combinado de crecimiento en telefonía residencial y celular da como resultante pasar de 811 mil teléfonos en 1994 a cerca de 8 millones en el 2005, lo que representa un salto de 9.6 veces.

\section{Comparación con los países latinoamericanos}

A pesar del notable crecimiento del sector telefónico, expresado en la densidad telefónica en el Perú las cifras al año 2003 -ya que no disponemos de estadísticas homogéneas para años posteriores- son más bien modestas si las comparamos con las tasas de crecimiento que han experimentado otros países del continente americano. Según datos de la Internacional Telecommunications Union (ITU), órgano rector de las telecomunicaciones en el mundo, al año 2003 -valga la redundancia- el Perú tenía las menores tasas de desarrollo telefónico de América del Sur

Gráfico 3

Densidad telefónica en el Perú (1994-2005)

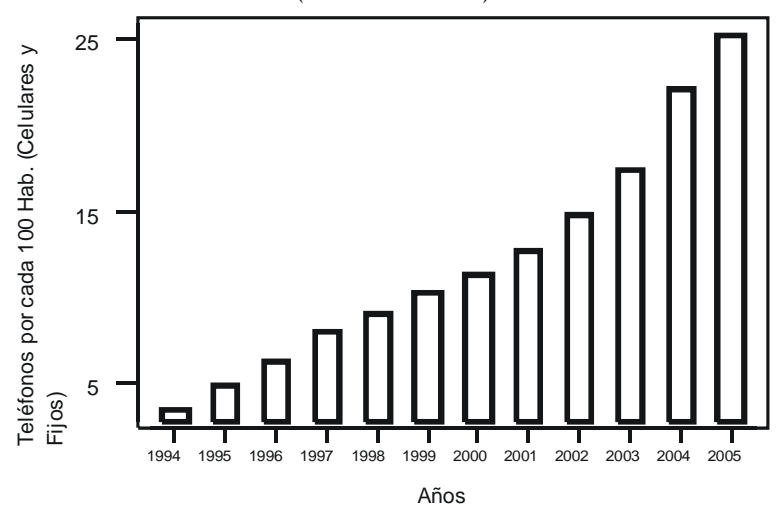

(Ver Gráfico 4). Con una densidad de 17.32, quedaba por debajo de Bolivia que tenía 23.81; y muy, pero muy debajo de nuestro vecino Chile que ostentaba 65.87 teléfonos por cada cien habitantes. Es decir, que Chile estaba casi cuatro veces más desarrollado que el Perú en esta materia (Ver Cuadro 2). En cuanto a telefonía fija, el Perú superaba solamente a Paraguay, y en telefonía celular nuestro país es el último de la tabla.

Es decir, del entusiasmo pasamos a la preocupación. Considerando que la base de partida de los diversos países en América Latina ha sido muy semejante ya que el proceso de la privatización del sector ocurrió casi en la misma época, las cifras peruanas deberían estar a la par de sus vecinos de la región. Pero, la situación tal vez refleja la existencia de un conjunto de variables que generan un orden de cosas restrictivo y que están frenando el desarrollo del sector en comparación con nuestros vecinos.

Se puede plantear a modo de hipótesis que tres hechos combinados constituyen las variables restrictivas y que podrían explicar el fenómeno de bajo crecimiento relativo de la telefonía en el Perú:

a. El casi monopolio de la concesionaria Telefónica del Perú en la operación de la telefonía residencial fija desde 1994 a la fecha. Esta compañía española es, en los hechos, el único operador a pesar que operan otros más. Su peso es tan grande que las demás no llegan al 8\% de participación combinada. El resultado de la falta de competencia es el alto costo del servicio telefónico residencial fijo. Una familia está pagando un promedio de US\$ 35.00 al mes por un servicio local que brinda unos 600 minutos gratuitos al

\section{Gráfico 4}

Densidad Telefónica en la Región Latinoamericana en el 2003.

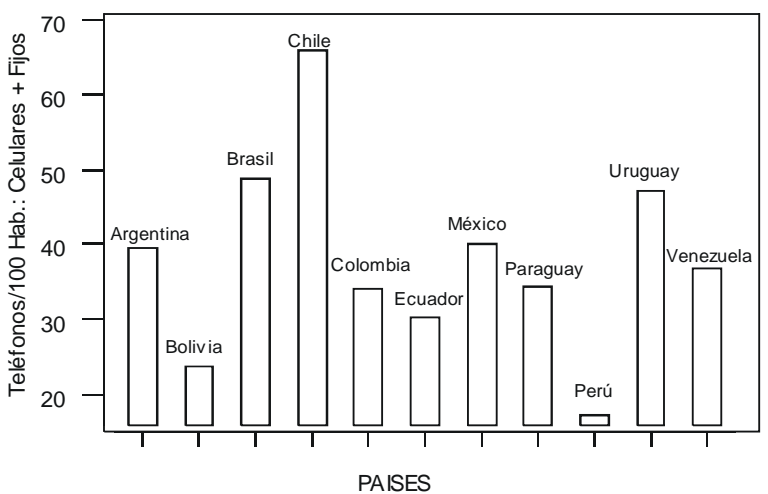




\section{Cuadro 1}

Cantidad de teléfonos fijos y celulares en el Perú.

\begin{tabular}{lllllll}
\hline AÑO & $\begin{array}{l}\text { LíNEAS } \\
\text { CELULARES }\end{array}$ & $\begin{array}{l}\text { LINEAS } \\
\text { TELÉFONOS }\end{array}$ & $\begin{array}{l}\text { TOTAL } \\
\text { TELÉFONOS }\end{array}$ & $\begin{array}{l}\text { DENSIDAD } \\
\text { CELULARES }\end{array}$ & $\begin{array}{l}\text { DENSIDAD } \\
\text { TELÉFONOS }\end{array}$ & $\begin{array}{l}\text { DENSIDAD } \\
\text { TELEFÓNICA }\end{array}$ \\
& & & & FJJOS & TOTAL \\
\hline $\mathbf{1 9 9 4}$ & 52,000 & 759,191 & 811,191 & 0.22 & 3.21 & 3.43 \\
$\mathbf{1 9 9 5}$ & 75,397 & $1,088,176$ & $1,163,573$ & 0.31 & 4.53 & 4.84 \\
$\mathbf{1 9 9 6}$ & 201,895 & $1,332,356$ & $1,534,151$ & 0.83 & 5.45 & $\mathbf{6 . 2 8}$ \\
$\mathbf{1 9 9 7}$ & 435,706 & $1,537,341$ & $1,973,047$ & 1.75 & 6.18 & $\mathbf{7 . 9 3}$ \\
$\mathbf{1 9 9 8}$ & 736,294 & $1,553,874$ & $2,290,168$ & 2.91 & 6.14 & $\mathbf{9 . 0 5}$ \\
$\mathbf{1 9 9 9}$ & $1,045,710$ & $1,609,884$ & $2,665,594$ & 4.06 & 6.26 & $\mathbf{1 0 . 3 2}$ \\
$\mathbf{2 0 0 0}$ & $1,339,667$ & $1,617,582$ & $2,957,249$ & 5.12 & 6.19 & $\mathbf{1 1 . 3 1}$ \\
$\mathbf{2 0 0 1}$ & $1,793,284$ & $1,570,956$ & $3,364,240$ & 6.76 & 5.92 & $\mathbf{1 2 . 6 8}$ \\
$\mathbf{2 0 0 2}$ & $2,306,943$ & $1,656,624$ & $3,963,567$ & 8.56 & 6.15 & $\mathbf{1 4 . 7 1}$ \\
$\mathbf{2 0 0 3}$ & $2,930,343$ & $1,839,165$ & $4,769,508$ & 10.71 & 6.72 & $\mathbf{1 7 . 4 3}$ \\
$\mathbf{2 0 0 4}$ & $4,092,558$ & $2,049,822$ & $6,142,380$ & 14.74 & 7.33 & $\mathbf{2 2 . 0 7}$ \\
$\mathbf{2 0 0 5}$ & $5,583,356$ & $2,250,921$ & $7,834,277$ & 20.51 & 8.27 & $\mathbf{2 8 . 7 8}$ \\
\hline
\end{tabular}

Fuente: Elaborado por el autor, basado en estadísticas de OSIPTEL. www.osiptel.gob.pe

Cuadro 2

Densidad Telefónica en los países de América del Sur (Año 2003)

\begin{tabular}{lcll}
\hline PAIS & $\begin{array}{l}\text { DENSIDAD EN } \\
\text { TELÉFONOS FIJOS }\end{array}$ & $\begin{array}{l}\text { DENSIDAD EN } \\
\text { CELULARES }\end{array}$ & $\begin{array}{l}\text { TOTAL } \\
\text { DENSIDAD }\end{array}$ \\
\hline Argentina & 21.88 & 17.76 & 39.64 \\
Bolivia & 7.14 & 16.67 & 23.81 \\
Brasil & 22.32 & 26.36 & 48.68 \\
Chile & 23.04 & 42.83 & 65.87 \\
Colombia & 20.03 & 14.13 & 34.16 \\
Ecuador & 11.91 & 18.41 & 30.32 \\
Paraguay & 4.61 & 29.85 & 34.46 \\
Perú & 6.71 & 10.61 & 17.32 \\
Uruguay & 27.96 & 19.29 & 47.25 \\
Venezuela & 11.27 & 25.64 & 36.91 \\
México & 14.67 & 25.45 & 40.12 \\
\hline
\end{tabular}

Fuentes: Internacional Telecommunications Union, y el National Bureau of Statistics de la República Popular China.

mes, lo que da unos 20 minutos por día. Ya hubieron varios intentos de parte de algunos líderes políticos o de defensa del consumidor para reducir dichas tarifas e implementar una tarifa plana o flat, pero la empresa ha demostrado una gran capacidad de reacción para neutralizar dichas iniciativas. El medio legal y técnico en que se ampara la empresa es el contrato de privatización que le brinda una serie de privilegios, entre ellos el derecho de facto a establecer las tarifas prohibitivas por el acceso al uso de su infraestructura de cableado y ductos nacionales. Dichas tarifas espantan cualquier asomo de competencia. A ello se suma la notoria debilidad del órgano supervisor (OSIPTEL), que tiene atribuciones mas bien formales que reales al respecto. Es cu- 
rioso, pero el funcionamiento de OSIPTEL se financia con una tasa especial que pagan las empresas telefónicas, lo que resulta en un obvio elemento vinculante del supervisor con el supervisado.

b. Una débil promoción gubernamental y privada del desarrollo de la telefonía rural. Es decir una imperceptible inversión estatal en aquellas regiones donde el inversionista privado no llega.

c. El alto costo por minuto de la telefonía celular, así como de los aparatos telefónicos celulares. Esto ocurre porque el número de operadores es bajo, siendo la competencia extremadamente limitada, lo que ha dado lugar a serios debates públicos sobre sospechas de concertación de precios entre operadores.

\section{Comparación con los países desarrollados}

Si comparamos nuestras estadísticas de desarrollo telefónico con las de países desarrollados, la situación revela un atraso aún mayor. La densidad telefónica para estos países ya no se mide por el número de teléfonos por cada cien habitantes, sino por habitante. Por ejemplo Suiza tiene 1.55 teléfonos por habitante, seguido por Alemania con 1.45, Inglaterra con 1.40, Estados Unidos con 1.15, y así sucesivamente (Ver Gráfico 5).

\section{Gráfico 5}

Densidad Telefónica en países desarrollados.

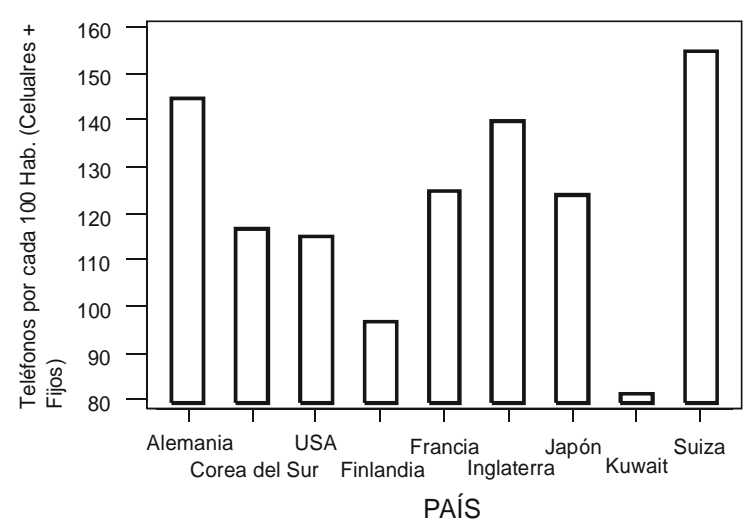

El crecimiento telefónico de los países desarrollados tiene una historia larga. Si comparamos las estadísticas del Perú con los Estados Unidos, leemos historias diferentes: el país norteamericano tiene una densidad telefónica significativa desde los años veinte, mientras que el Perú en esta materia está asomando recién a inicios del presente siglo. Resulta que la densidad telefónica del Perú en el año 2005 es la que este país tenía en 1955; es decir, de hace de cincuenta años atrás, y en la actualidad estamos en el $22 \%$ del progreso de dicho país. Es decir, a pesar de todo el progreso, la brecha telefónica persiste. (Ver Cuadro 3).

\section{Cuadro 3}

Evolución de la densidad telefónica Perú vs. Estados Unidos.

\begin{tabular}{lcc}
\hline AÑO & $\begin{array}{l}\text { DENSIDAD } \\
\text { TELEFÓNICA } \\
\text { ESTADOS UNIDOS }\end{array}$ & $\begin{array}{l}\text { DENSIDAD } \\
\text { TELEFÓNICA } \\
\text { PERÚ }\end{array}$ \\
\hline $\mathbf{1 9 2 0}$ & 9.6 & $\mathrm{~s} / \mathrm{d}$ \\
$\mathbf{1 9 3 0}$ & 12.5 & $\mathrm{~s} / \mathrm{d}$ \\
$\mathbf{1 9 4 0}$ & 12.7 & $\mathrm{~s} / \mathrm{d}$ \\
1950 & 21.7 & $\mathrm{~s} / \mathrm{d}$ \\
1960 & 27.6 & $\mathrm{~s} / \mathrm{d}$ \\
1970 & 35.0 & $\mathrm{~s} / \mathrm{d}$ \\
1980 & 44.8 & $\mathrm{~s} / \mathrm{d}$ \\
1990 & 54.3 & 3.4 \\
$\mathbf{2 0 0 0}$ & 66.4 & 11.3 \\
$\mathbf{2 0 0 5}$ & $115.0(*)$ & 28.8 \\
\hline
\end{tabular}

Fuente: Elaborado por el autor basado en estadísticas de OSIPTEL (www.osiptel.gob.pe), de FCC (www.fcc.gov), y del World Factbook (www.cia.gov).

\section{La cobertura de telefonía a nivel nacional}

La cobertura telefónica al interior del Perú es dispareja: más bien resulta chocante por su desigualdad. De los casi ocho millones de teléfonos entre fijos y celulares que tiene el Perú, más de cinco millones, o el $64 \%$, se encuentran en Lima, casi las dos terceras partes del total. Le siguen en importancia La Libertad, Arequipa y Lambayeque con cifras que están por debajo del 5\% del total. (Ver Cuadro 4).

Si analizamos la densidad telefónica descubrimos el mismo fenómeno. De un promedio nacional que llega a 28.8 teléfonos por cada cien habitantes, Lima y Callao tienen una densidad que supera el 55.95. En cambio, en el otro extremo, muchos departamentos de la sierra y de la selva tienen densidades de escándalo. Es el caso de Huancavelica, el departamento más pobre del Perú, que llega a 1.63 teléfonos por cada cien habitantes. Otros no están muy lejos como Amazonas con 3.69, Apurímac con 4.30, Pasco con 5.94, San Martín con 5.94. (Ver Gráfico 6).

En el medio de ambos extremos hay un conjunto de departamentos con densidad intermedia como Arequipa con 35.95, Tacna con 35.63, 


\section{Gráfico 6}

Densidad Telefónica en el Perú al 2005.

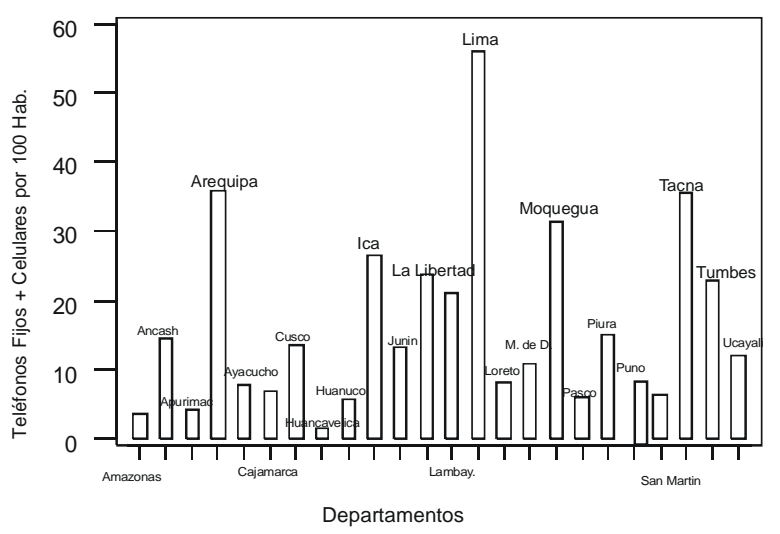

Moquegua con 31.50; así como Ica, La Libertad, Tumbes y Lambayeque con cifras superiores a veinte.

Hay que resaltar que entre los departamentos con menor densidad telefónica se encuentran la mayoría de los fronterizos como Amazonas, Loreto, Cajamarca, Puno, Madre de Dios y Ucayali. Esta concentración no es privativa de la telefonía fija residencial sino también de la celular.

En conclusión, el poder de comunicación telefónica está concentrado en la capital de la república, y en unos pocos departamentos con vibración modernizadora y que son espacios del desarrollo agroindustrial y/o minero.

Cuadro 4

Cobertura telefónica nacional por Departamentos al 2005.

\begin{tabular}{|c|c|c|c|c|c|c|}
\hline Departamento & $\begin{array}{l}\text { Teléfonos } \\
\text { Fijos }\end{array}$ & $\begin{array}{l}\text { Teléfonos } \\
\text { Celulares }\end{array}$ & Población & $\begin{array}{l}\text { Densidad } \\
\text { Telf. Fijos }\end{array}$ & $\begin{array}{l}\text { Densidad } \\
\text { Celular }\end{array}$ & $\begin{array}{l}\text { Densidad } \\
\text { Total }\end{array}$ \\
\hline Amazonas & 6,116 & 10,512 & 450,538 & 1.36 & 2.33 & 3.69 \\
\hline Ancash & 54,543 & 112,382 & $1,154,523$ & 4.72 & 9.73 & 14.46 \\
\hline Apurímac & 6,569 & 14,322 & 485,934 & 1.35 & 2.95 & 4.30 \\
\hline Arequipa & 104,460 & 305,259 & $1,139,599$ & 9.17 & 26.79 & 35.95 \\
\hline Ayacucho & 13,551 & 31,840 & 581,656 & 2.33 & 5.47 & 7.80 \\
\hline Cajamarca & 29,096 & 79,416 & $1,550,132$ & 1.88 & 5.12 & 7.00 \\
\hline Cusco & 44,135 & 119,301 & $1,252,201$ & 3.52 & 9.52 & 13.05 \\
\hline Huancavelica & 3,169 & 4,444 & 468,161 & 0.68 & 0.95 & 1.63 \\
\hline Huanuco & 13,418 & 35,454 & 844,649 & 1.59 & 4.20 & 5.79 \\
\hline Ica & 47,840 & 143,165 & 720,691 & 6.64 & 19.86 & 26.50 \\
\hline Junín & 54,674 & 116,822 & $1,288,792$ & 4.23 & 9.06 & 13.29 \\
\hline La Libertad & 117,914 & 254,580 & $1,573,106$ & 7.50 & 16.18 & 23.68 \\
\hline Lambayeque & 71,614 & 171,712 & $1,151,411$ & 6.22 & 14.91 & 21.13 \\
\hline Loreto & 31,127 & 46,684 & 943,807 & 3.30 & 4.95 & 8.24 \\
\hline M. de Dios & 3,100 & 8,656 & 107,644 & 2.88 & 8.04 & 10.92 \\
\hline Moquegua & 10,628 & 42,060 & 167,251 & 6.35 & 25.15 & 31.50 \\
\hline Pasco & 4,869 & 11,968 & 283,649 & 1.72 & 4.22 & 5.94 \\
\hline Piura & 74,030 & 184,517 & $1,710,790$ & 4.33 & 10.79 & 15.11 \\
\hline Puno & 23,010 & 95,854 & $1,313,571$ & 1.75 & 7.30 & 9.05 \\
\hline San Martín & 21,843 & 26,862 & 788,195 & 2.77 & 3.41 & 6.18 \\
\hline Tacna & 21,292 & 91,882 & 317,619 & 6.70 & 28.93 & 35.63 \\
\hline Tumbes & 9,901 & 39,287 & 215,634 & 4.59 & 18.22 & 22.81 \\
\hline Ucayali & 16,943 & 39,184 & 468,922 & 3.61 & 8.36 & 11.97 \\
\hline Lima y Callao & $1,420,136$ & $3,597,193$ & $8,968,279$ & 15.84 & 40.11 & 55.95 \\
\hline TOTAL & $2,250,921$ & $5,583,356$ & $27,946,774$ (*) & 7.89 & 19.98 & \\
\hline
\end{tabular}

Fuentes: OSIPTEL y Perú en Números de «Cuánto».

(*) Calculado sobre la base de la proyección poblacional de Perú en Números 2005. 


\section{Los operadores telefónicos en el Perú}

Los operadores de telefonía en el Perú los podemos clasificar en cuatro categorías:

- Operadores de Telefonía Fija

- Operadores de Telefonía Celular

- Operadores de Telefonía a Larga Distancia

- Operadores de Telefonía Pública

En cada una de dichas categorías el principal operador es Telefónica del Perú.

- Operadores en telefonía fija. En telefonía residencial fija hay, como se dijo, una concentración monopólica de Telefónica del Perú (TdP) con el $96 \%$ de participación del mercado (2’156,638 líneas) (ver Gráfico 7). Las otras empresas como Telmex, Comunicaciones Móviles (ex Bellsouth), Americatel e Impsat, que cuentan en conjunto con 47,310 líneas, operan no utilizando las instalaciones de cableado y ductos de Telefónica del Perú sino tecnologías alternas, mayormente de tipo radial. Ese es el caso de Bellsouth que lanzó al mercado un teléfono inalámbrico de pared o de consola pero que tuvo muy baja acogida debido al elevado precio por minuto, que resultaba siendo muy cercano al costo del teléfono celular. Bellsouth fue absorbida en el 2004 por TdP.

- Operadores de telefonía celular. En telefonía celular también existe una clara concentración en un duopolio. Una vez más, TdP tiene un amplísimo dominio del mercado con el $61 \%$ de participación (3’383,514 abonados). La española es seguida por Claro -antes TIMcon un 34\% (1'948,591 clientes), y en un lugar minoritario, con tendencia a la baja, se ubica

Gráfico 7. Cantidad de teléfonos fijos por empresa operadora

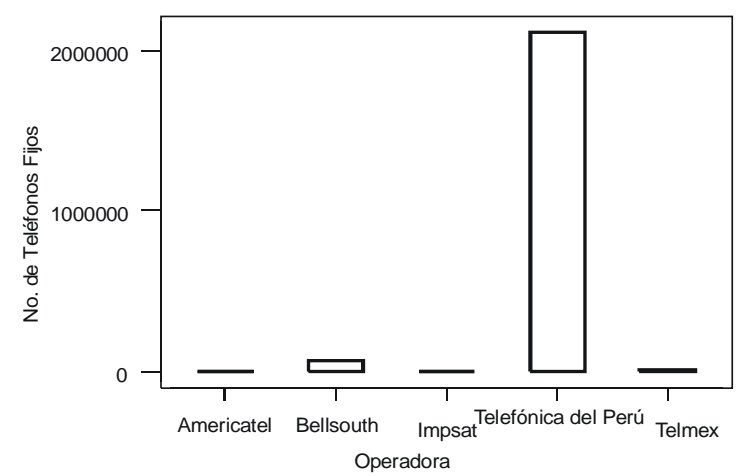

Comunicaciones Móviles con 5\% de participación (251,251 aparatos).

- Operadores en telefonía a larga distancia. A diferencia de la escasa oferta en telefonía fija y celular, en telefonía a larga distancia existían dos docenas de operadores en el 2005, con una oferta variada y atractiva; sin embargo, la de mayor movimiento corresponde, una vez más, a TdP, con un $54 \%$ de la participación en las llamadas salientes $(42,289)$. Le sigue IDT con un lejano $13 \%(10,427$ llamadas). Las demás operadoras tienen cifras por debajo del $5 \%$ o no significativas.

- Operadores de telefonía pública. La telefonía pública también ha experimentado un crecimiento importante (Ver Gráfico 8). De ocho mil teléfonos públicos de los que disponían los peruanos en 1993, hemos pasado a cerca de 150 mil en septiembre del 2005, lo que da un crecimiento de dieciocho veces en el período de doce años, con una densidad que ha ascendido en dicho periodo de 0.35 a 5.23 teléfonos públicos por cada mil habitantes. Por cuarta vez, el mayor y casi monopólico operador de telefonía pública del Perú es, como se podrá apreciar una vez más, TdP con cerca del $90 \%$ de participación. No obstante, la lenta apertura de la competencia en la telefonía pública en el Perú está llevando a una disminución de la tarifa por minuto. Así, mientras que TdP sigue cobrando un sol el minuto por llamadas a nivel nacional, la empresa mexicana Telmex cobra la mitad. Pero, el número de teléfonos públicos de Telmex está en un estado germinal, tiene menos de cinco mil teléfonos frente a los más de 133 mil que tiene la empresa española repartidos a nivel nacional.

Una observación final: la tendencia al crecimiento del número de teléfonos públicos no es ya un fenómeno aplicable a los países desarrollados, pues ocurre que en Estados Unidos el número de teléfonos públicos ha decrecido de 2’086,540 en 1997 a 1'495,786 en el 2003', con una caída de $30 \%$ en seis años. Esto, evidentemente, tiene correlación con la mayor disponibilidad de teléfonos celulares lo que ha traído como consecuencia un menor uso de los teléfonos públicos, y por lo tanto una disminución de su rentabilidad, y un retiro de las calles. 
Puede deducirse que el crecimiento de la oferta de telefonía pública en el Perú es el reflejo de la aún baja densidad telefónica que hay en el país. Es probable que en la medida que aumente dicho indicador, hasta llegar a una cifra crítica, empiece a disminuir la velocidad de crecimiento de los teléfonos públicos. Es de esperar que antes de que esa desaceleración ocurra, el gobierno decida actuar con urgencia para dirigir la instalación de la oferta de teléfonos públicos en las zonas rurales de sierra y selva, y en los pueblos menores de tres mil habitantes. Hay que estar advertidos que si se deja al libre mercado la instalación de telefonía pública, la desaceleración, freno o disminución de teléfonos público podría ocurrir sin haber logrado conectar a todas las comunidades rurales del país por este medio.

\section{CONCLUSIONES}

1. Existe una explosión mundial en el uso del teléfono. Esto se debe a varios factores, entre ellos: mejora de la tecnología de comunicaciones, uso de la fibra óptica y disminución del costo por minuto, tanto en llamadas locales como en llamadas internacionales.

2. Entre 1994 y 2005, el Perú ha crecido en densidad telefónica de 3.8 a 28.8 teléfonos por cada cien habitantes. A pesar de este significativo avance, el Perú tiene el menor índice de penetración telefónica en la región: está casi al mismo nivel de Bolivia, pero más de tres veces por debajo de Chile.

3. La región sudamericana está muy lejos de las regiones desarrolladas del mundo. Así mientras nosotros contamos con 26 teléfonos por cada cien habitantes, en países como Suiza la relación es de 155, en Alemania 145, en Inglaterra 140, y en Estados Unidos 115 teléfonos por cada cien habitantes. Esto quiere decir que la brecha tecnológica con los países desarrollados se mantiene intacta. Hemos progresado como país, pero ellos lo han hecho mucho más.

4. Internamente el Perú tiene un desarrollo heterogéneo: Lima, la capital, tiene una densidad de 56 teléfonos por cada cien habitantes, mientras que en el otro extremo tenemos regiones como Huancavelica que tiene una densidad de solamente 1.6.
5. Entre los factores limitantes al desarrollo telefónico que se observan en el Perú tenemos: legislación y actitud del Estado complaciente con la presencia casi monopólica de una sola empresa operadora que es Telefónica del Perú, con sede matriz en España.

6. Al año 2005 , TdP tenía el $96 \%$ de la telefonía fija residencial, el 63\% de telefonía celular, un $54 \%$ de las llamadas salientes en telefonía de larga distancia y un $90 \%$ en telefonía pública. Su competencia más cercana está conformada por Claro con el $32 \%$ en telefonía celular, e IDT con 13\% en llamadas a larga distancia.

7. A esto se suma la escasa promoción del rol del Estado para penetrar en los sectores rurales.

\section{NOTAS}

1 National Bureau of Statistics. People's Republic of China. Página web.

2 Federal Communications Comission. Ver www.fcc.gov.

3 Decreto Supremo №11-94-TC del 13 de Mayo de 1994. Contrato de Concesión de ENTEL Perú y Compañía Peruana de Teléfonos S.A.

4 Ver $w w w \cdot f c c . g o v$.

\section{BIBLIOGRAFIA}

Agencia Central de de Inteligencia. Estados Unidos. Página web: www.cia.gov.

Cuánto Publicaciones. (2005). Perú en Números. Lima.

Federal Communications Comisión. Estados Unidos. Página web: $w w w . f c c . g o v$.

Gibson, Stephen W. (1987). Cellular Mobile Radiotelephones. Englewood Cliff: Prentice Hall.

Gross, Lynne Schafer. (1983). Telecommunications. An Introduction to Radio, Television and Other Electronic Media. Segunda Edición. Wcb. Dubuque, Iowa.

Instituto Nacional de Estadística e Informática. Perú. Página web: www.inei.gob.pe.

International Telecommunications Union. Página web: www.itu.org.

Library of Congress. Estados Unidos. Página web: www.loc.gov. 
National Bureau of Statistics. República Popular China. Página web: www.nbs.gov.cn.

Organismo Supervisor de la Inversión Privada en Telecomunicaciones del Perú (OSIPTEL). (1996). Marco Legal de las Telecomunicaciones y Contratos de Concesión de Telefónica del Perú. Lima.
Organismo Supervisor de la Inversión Privada en Telecomunicaciones del Perú (OSIPTEL). Página web: www.osiptel.gob.pe

Telefónica del Perú SAA. Página web: www.telefonica.com.pe. 